\title{
Assessing the extent of SARS-CoV-2 circulation through serological studies
}

\author{
The COVID-19 pandemic has highlighted the crucial importance of antibody surveys in estimating the incidence \\ of SARS-CoV-2 infection at a population level. Studies in Brazil and China address the use of serology to obtain a \\ better estimate of the infection rate.
}

\section{Marion Koopmans and Bart Haagmans}

E stimates of the spread of the coronavirus SARS-CoV-2 and the severity of COVID-19 disease based on the reporting of disease symptoms overestimate the true disease severity if people with mild or asymptomatic infection are not included in the case findings, and they also underestimate the prevalence of disease. Serology surveys in which levels of antibodies raised against the virus are assessed in people have the potential to provide a more comprehensive picture of who has been infected ${ }^{1-3}$ (Fig. 1).

Studies of households of patients with COVID-19 and passengers on the Diamond Princess cruise ship and other outbreak investigations provided the first evidence of asymptomatic infections and suggestive evidence for their role in transmission ${ }^{4}$. However, a question that remains is the extent to which such people remain asymptomatic or if they developed symptoms after the time point of testing (presymptomatic). For this, carefully designed prospective cohort studies are needed that include clinical follow-up as well as repeated biological sampling to test for presence of the virus and for antibodies after infection. A study involving more than $80 \%$ of the citizens of the village of Vo' in northern Italy and a large population-wide study in Iceland confirmed the presence of truly asymptomatic viral shedders ${ }^{5,6}$.

To understand the true incidence of SARS-CoV-2 infection, community-representative population studies are required to allow a more accurate estimation of all people infected. A first prerequisite for the use of serology to measure the extent of circulation is understanding the kinetics of antibody development in SARS-CoV-2-infected people. There is a growing body of literature providing evidence that people with COVID-19 develop antibodies that are detectable in the majority of people 3-4 weeks after symptom onset, although the antibody levels are lower and may be delayed

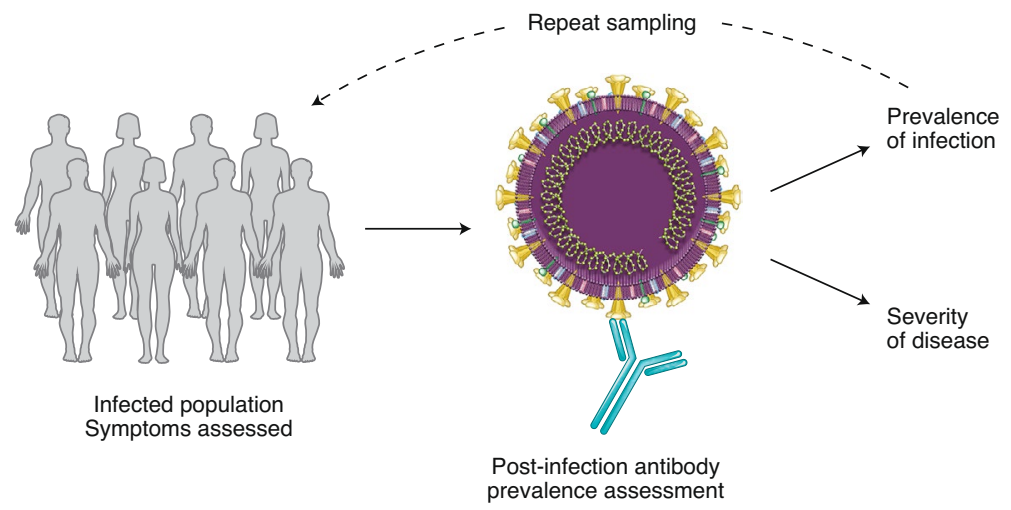

Fig. 1 Antibody testing to assess the extent of SARS-CoV-2 circulation. Serology surveys that are able detect antibodies after SARS-CoV-2 infection have the potential to identify those who were asymptomatic and were not identified by symptom reporting and hence provide a more complete picture of the prevalence and severity of disease. Repeating these tests in populations further deepens the understanding of the stage of the pandemic in this population.

in people with milder symptoms ${ }^{1}$. However, the kinetics of these changes are unknown for people with asymptomatic infection.

Long et al. describe a cohort of people in Chongquing province, China, who were diagnosed during routine RT-PCR screening following guidance from contact tracing ${ }^{1}$. These people did not have any symptoms 2 weeks before diagnosis or during follow-up. In accordance with the local infection-control strategy, patients were quarantined in a dedicated facility, were monitored for the development of symptoms, and were repeatedly assessed for the presence of the virus or antibodies to the virus by RT-PCR and antibody testing. The authors found that almost $20 \%$ of these asymptomatic people did not have detectable IgG antibodies 3-4 weeks later, that their levels of antibodies were lower than those in symptomatic people around the same time, and that the antibody levels started to decline rapidly during the 'early convalescent phase' (the term used by the authors, although the people were considered asymptomatic). One factor that may have contributed to the rapid decline is that even asymptomatic people in quarantine received a range of antiviral treatments that may have affected the development of an immune response, and thus this was not reflective of what happens during an infection without such interventions.

Nevertheless, the observations from the paper suggest that a proper estimation of the number of people who are infected, including those who do not become ill (the infection attack rate), needs to be carefully timed. Here, the choice of assays matters: after coronavirus infection, patients develop different types of antibodies to several viral proteins that may exert different antiviral effects ${ }^{7}$. The gold-standard assay for measuring functional antibodies assesses interference of the antibodies with the ability of the virus to replicate in cell culture. Although correlates of protection remain to be determined, such neutralizing antibodies (directed at the viral spike protein) are considered important. Long et al. found antibodies in $84 \%$ of people 
with symptomatic COVID-19 ${ }^{1}$, which suggests their assay was not very sensitive. Careful assay validation and the use of standards to allow comparison of results across serological surveys is needed to allow direct comparisons of data from different studies $^{7,8}$. An alternative explanation could be that the people received a mixture of antiviral treatments, which may have affected their development of an immune response ${ }^{1}$.

$\mathrm{Xu}$ et al. describe the first large-scale serosurvey from Wuhan and other provinces in China, carried out between early March and April 2020, when the number of new cases of COVID-19 started to decline ${ }^{2}$. The authors made an effort to study seroprevalence in different regions, focusing on hospital settings and community settings. They used the same assay as that described by Long et al. ${ }^{1}$ and found that the prevalence of IgG antibodies ranged from $0.4 \%$ in healthcare workers in Guangdong province to $3.8 \%$ in those from Wuhan in Hubei province. Relatives of healthcare workers in Wuhan and hotel staff had a seroprevalence similar to that of the healthcare workers, whereas the antibody prevalence in community residents and factory workers in Sichuan and Guangdong was lower. The study design precludes the drawing of conclusions about the differences, but the overall message confirms what has been observed elsewhere: although the healthcare system was overwhelmed by severe cases, the vast majority of the population did not show evidence of infection. Therefore, continued vigilance will be needed to monitor circulation of the virus and to control outbreaks before they get out of control.

Silveira et al. describe a repeated population 'snapshot' in which members of randomly selected households were tested for antibodies to SARS-CoV-2 ${ }^{3}$. The surveys were done in the state of Rio Grande do Sul in Brazil during two rounds in April 2020. By 30 April, the state had 50 recorded deaths and therefore this could be considered a very early estimate of the infection attack rate in the region. The authors found seroprevalence rates to be well below $0.5 \%$, although the antibody prevalence in household contacts of confirmed cases was considerably higher. In this study, a rapid seroprevalence test was used.

Containing the spread of SARS-CoV-2 has proven to be a huge challenge, stretching capacity of healthcare systems and public health at great cost to society and the economy. The three studies here confirm that the world is most likely in the early stages of this pandemic ${ }^{2,3}$, that the majority of the population remains susceptible to infection, and that antibodies may wane quite rapidly ${ }^{1}$. Serosurveys combined with in-depth outbreak investigations to elucidate spread of the virus and cohort studies aimed at delineating the kinetics of infection and immune responses provide critical input for epidemic forecasting models ${ }^{9}$. For seasonal human coronaviruses, mucosal immunity is thought to be short-lived, although the data are mostly circumstantial and the role of repeated infections in development of cross-reactive antibodies remains to be clarified $^{10,11}$. Another important need is the use of standards to allow validation and comparison of data generated by different assays and platforms for serology ${ }^{8}$. This is needed to provide an independent reference for the development of commercial platforms, the evaluation of antibody-based treatments, and the evaluation of vaccine responses ${ }^{7}$.

In the context of immunity, serosurveys skim the surface; they are a proxy for a much broader response to infection that may lead to immunity. Full understanding of immunity requires knowledge about differences in susceptibility to infection, innate immunity, the quality and functionality of antibodies, and the involvement of B cells and T cells in people with different severities of illness, sex, ethnicity, and ages. Key outstanding questions are the longevity of the immune responses, correlates of protection, the role of pre-existing immunity from exposure to seasonal coronaviruses, and whether or not there is potential for antibody-dependent enhancement of disease ${ }^{12}$. The surveys here provide a beginning to answering these questions.

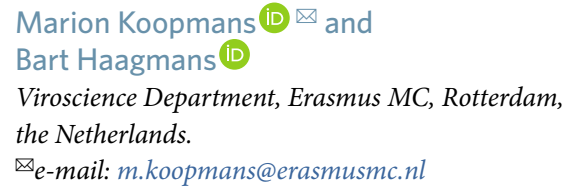

2. Xu, X. et al. Nat. Med. https://doi.org/10.1038/s41591-020-0949-6 (2020).

3. Silveira, M.F. et al. Nat. Med. https://doi.org/10.1038/s41591-0200992-3 (2020).

4. Oran and Topol. Ann. Intern. Med. https://doi.org/10.7326/M203012 (2020).

5. Lavezzo, E. et al.. Nature https://doi.org/10.1038/s41586-0202488-1 (2020).

6. Gudbjartsson, D.F. et al. N. Engl. J. Med. https://doi.org/10.1056/ NEJMoa2006100 (2020).

7. GeurtsvanKessel, C.H. et al. Nat. Commun. 11, 3436 (2020).

8. Leung, G. M. et al. Epidemiol. Infect. 134, 211-221 (2006).

9. Kissler, S. M., Tedijanto, C., Goldstein, E., Grad, Y. H. \& Lipsitch, M. Science 368, 860-868 (2020).

10. Edridge, A.W.D. et al. medRxiv https://doi.org/10.1101/ 2020.05.11.20086439 (2020).

11. Ou, X. et al. Nat. Commun. 11, 1620 (2020).

12. Coish, J.M. \& MacNeil, A.J. Microbes Infect. https://doi.org/ 10.1016/j.micinf.2020.06.006 (2020).

\title{
Bringing monogenic disease screening to the clinic
}

\author{
The Healthy Nevada Project shows that otherwise invisible disease risk can be revealed through DNA-based screening. \\ Identifying these monogenic risks could be the first step toward a new population health-screening program.
}

Michael F. Murray and Monica A. Giovanni

T he promise of DNA-based screening is that rather than waiting for a disease such as cancer or heart disease to affect a person or their family member, disease risk can be identified in a person through screening so that preventive interventions can be offered prior to disease. This new mode of risk ascertainment has the predicted but as-yet unproven ability to act at the population level to prevent more relevant disease associated with US Centers for Disease Control (CDC) Tier 1 conditions than current approaches $\mathrm{do}^{1,2}$. The CDC Tier 1 conditions include two different cancer syndromes (hereditary breast and ovarian 